\title{
The role of oxidative stress in the development of pulmonary arteriovenous malformations after cavopulmonary anastomosis
}

Sunil P. Malhotra, MD

V. Mohan Reddy, MD

Stephan Thelitz, MD

You-Ping $\mathrm{He}, \mathrm{PhD}$

D. Michael McMullan, MD*

Frank L. Hanley, MD

R. Kirk Riemer, PhD

From the Division of Cardiothoracic Surgery, University of California, San Francisco, Calif.

Sunil P. Malhorta is a recipient of the National Research Service Award (F32 HL 10339-01) from the National Heart, Lung, and Blood Institute of the National Institutes of Health.

Received for publication May 14, 2001; revisions requested Aug 3, 2001; revisions received Sept 10, 2001; accepted for publication Sept 18, 2001.

Read at the Eighty-first Annual Meeting of The American Association for Thoracic Surgery, San Diego, Calif, May 6-9, 2001.

Address for reprints: R. Kirk Riemer, PhD, Stanford University, Department of Cardiothoracic Surgery, Falk CVRB-CV116C, Stanford, CA 94305-5407 (E-mail: Riemerk@Stanford.edu).

*Present address: Department of Surgery, University of Texas, Houston, Tex.

J Thorac Cardiovasc Surg 2002;124:479-85

Copyright ( $(2) 2002$ by The American Association for Thoracic Surgery

0022-5223/2002 $\$ 35.00+0 \quad \mathbf{1 2 / 6 / 1 2 0 3 4 6}$

doi: $10.1067 / \mathrm{mtc} .2002 .120346$
Background: Cavopulmonary anastomosis is used for palliation of cyanotic heart disease. Clinically significant pulmonary arteriovenous malformations occur in up to $25 \%$ of patients after surgical intervention. Cavopulmonary anastomosis creates several modifications to pulmonary physiology that may contribute to the development of pulmonary arteriovenous malformations, including reduced pulmonary blood flow and the exclusion of inferior vena caval effluent.

Objective: By comparing the expression of angiogenic and stress-related proteins after cavopulmonary anastomosis and pulmonary artery banding, we sought to determine which genes were upregulated independent of reduced pulmonary blood flow.

Methods: Lambs aged 35 to 45 days were placed into 1 of 3 groups: cavopulmonary anastomosis $(\mathrm{n}=6)$, pulmonary artery banding $(\mathrm{n}=6)$, and sham control $(\mathrm{n}=6)$ animals. In our model pulmonary arteriovenous malformations are detectable by means of bubble-contrast echocardiography 8 weeks after cavopulmonary anastomosis. Lung tissue was harvested for Western blotting at 2 and 5 weeks after surgery.

Results: Cavopulmonary anastomosis and pulmonary artery banding both increased angiogenic gene expression, but only cavopulmonary anastomosis induced the expression of endothelial stress-related genes. Vascular endothelial growth factor was upregulated 2.5 -fold after both cavopulmonary anastomosis $(P=.002)$ and pulmonary artery banding $(P=.007)$. Only cavopulmonary anastomosis upregulated 2 stress-related genes, $\mathrm{HOl}$ and GLUT1, 2.7-fold $(P=.002)$ and 3.8 -fold $(P=.03)$, respectively. Hypoxia-inducible factor was upregulated 4-fold $(P=.003)$ after cavopulmonary anastomosis. Pulmonary artery banding failed to induce the increased expression of any of these proteins.

Conclusions: Reduced pulmonary blood flow induces a pulmonary angiogenic response but not an endothelial stress response. These results suggest that oxidative stress is more relevant to the formation of pulmonary arteriovenous malformations than angiogenic signaling alone because pulmonary artery banding does not result in pulmonary arteriovenous malformations. Oxidative stress of the pulmonary endothelium resulting from cavopulmonary anastomosis may predispose the affected vasculature to arteriovenous shunting. 


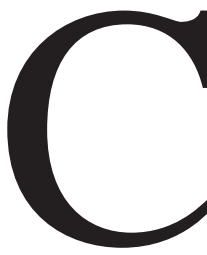

avopulmonary anastomosis (CPA) is performed for the palliation of tricuspid atresia and univentricular congenital heart defects. This procedure, as originally described by Glenn in 1958, uses an anastomosis between the superior vena cava (SVC) and the right pulmonary artery (RPA) to augment pulmonary blood flow. ${ }^{1}$ However, pulmonary arteriovenous malformations (AVMs) develop in a significant proportion of patients undergoing CPA. Several series have demonstrated clinically significant AVMs in up to $25 \%$ of patients after CPA. ${ }^{2,3}$ Sequelae of these pulmonary AVMs can range in severity from borderline hypoxemia to progressive cyanosis. To study this phenomenon, we have developed an ovine model that reliably produces pulmonary arteriovenous shunting 8 weeks after construction of the CPA.

Prior observations have demonstrated widespread pulmonary vascular remodeling after CPA. Histologic analyses of lung tissue after CPA reveal the proliferation of dilated microvascular structures. ${ }^{4,5}$ Recent findings from this animal model suggest that inhibited pulmonary angiotensinconverting enzyme expression and angiotensin II production may contribute to the chronic pulmonary vasodilation after CPA. ${ }^{6}$

Unfortunately, the physiologic and molecular basis for this abnormal pulmonary vascular remodeling remains poorly understood. CPA results in a number of potentially relevant modifications to the pulmonary circulation. Exclusion of inferior vena caval effluent is likely the most significant consequence of CPA. Another major physiologic alteration caused by CPA is reduced pulmonary blood flow. In this study we examined the pulmonary expression of oxidative stress-inducible and angiogenic proteins after CPA using our ovine model. To exclude changes in gene expression caused by reduced pulmonary blood flow, we performed similar analysis on age-matched lambs that underwent pulmonary artery banding (PAB). Because PAB does not result in the formation of pulmonary AVMs, changes in patterns of gene expression specific to CPA will yield valuable insight into the molecular mechanisms responsible for the development of such malformations.

This study examines the role of hypoxia-inducible factor $1 \alpha$ (HIF- $1 \alpha)$ in the development of pulmonary arteriovenous shunting. HIF- $1 \alpha$ has been implicated in the pathophysiology of several pulmonary vascular disorders, including pulmonary hypertension. ${ }^{7}$ HIF- $1 \alpha$ upregulates the expression of numerous genes involved in vascular remodeling by binding to a consensus nucleotide sequence known as the HIF- $1 \alpha$-responsive element. ${ }^{8}$ Some of these genes include those coding for the oxidative stress-associated factors hemoxygenase 1 (HOl) and glucose transporter 1 (GLUT1), as well as the angiogenic gene vascular endothelial growth factor $(V E G F)$ and its receptor, Flt-1. This study examines the early pulmonary expression (at 2 and 5 weeks after the operation) of these HIF- $1 \alpha$-inducible genes, as well as P-selectin (CD62), an HIF-1 $\alpha$-independent marker of endothelial activation, after $\mathrm{CPA}$ and $\mathrm{PAB}$.

\section{Methods}

\section{Animal Model}

Western sheep, aged 35 to 45 days ( $n=6$ ), were anesthetized with isoflurane (2\%), intubated, and mechanically ventilated. After median sternotomy and pericardiotomy, the SVC and RPA were identified and dissected free from their attachments. The RPA was divided near the pulmonary bifurcation, and the proximal end was oversewn (Figure 1). The SVC was similarly divided at the cavoatrial junction, and the atrial end was oversewn. Intravenous heparin was administered $(300 \mathrm{U} / \mathrm{kg}$ ), and $16 \mathrm{~F}$ to $20 \mathrm{~F}$ venous cannulas were placed to bypass the SVC to the right atrium. The SVC was then anastomosed to the RPA in an end-to-end fashion with running polypropylene sutures. The venous cannulas were removed, and the sternum was closed. After skin closure, the lambs were extubated and allowed to recover.

Another set of age-matched lambs underwent PAB $(n=6)$. After sternotomy, the left pulmonary artery (LPA) was dissected free from its attachments. The LPA was then encircled with umbilical tape, and an ultrasonic flow probe (Transonic Systems, Inc, Ithaca, NY) was placed. Once baseline flow measurements were recorded, the umbilical tape was tightened around the LPA with surgical clips to reduce flow to $20 \%$ of baseline values. The band was then further secured by 5-0 polypropylene sutures placed in the adventitia of the LPA.

A third group of lambs of the same age served as shamoperated control animals $(n=6)$. The preparation and dissection was the same as that described for the Glenn cohort. The RPA was occluded with a vascular clamp for 30 minutes. After this time, the clamp was removed, and the chest was closed as described above.

All animals received humane care in compliance with the "Principles of Laboratory Animal Care," formulated by the National Society of Medical Research, and the "Guide for the Care and Use of Laboratory Animals," prepared by the Institute of Laboratory Animal Resources, National Research Council, and published by the National Academy Press, revised 1996. The protocol was approved by the Committee on Animal Research at the University of California, San Francisco.

\section{Echocardiography}

Pulmonary AV shunting was demonstrated by means of bubble contrast echocardiography. Epicardial echocardiography was performed on each animal after median sternotomy. In the CPA cohort $5 \mathrm{~mL}$ of a saline solution-blood mixture was agitated to produce bubbles and then injected sequentially into the SVC and inferior vena cava. In the PAB group a similar bubbled saline solution-blood mixture was injected into each pulmonary artery sequentially. The appearance of bubbles in the left atrium within 5 cardiac cycles after SVC injection indicates AV shunting. ${ }^{9}$

\section{Tissue Harvest}

Lambs were killed at 2 and 5 weeks after the operation to obtain tissue for analysis. Animals were intubated and mechanically 


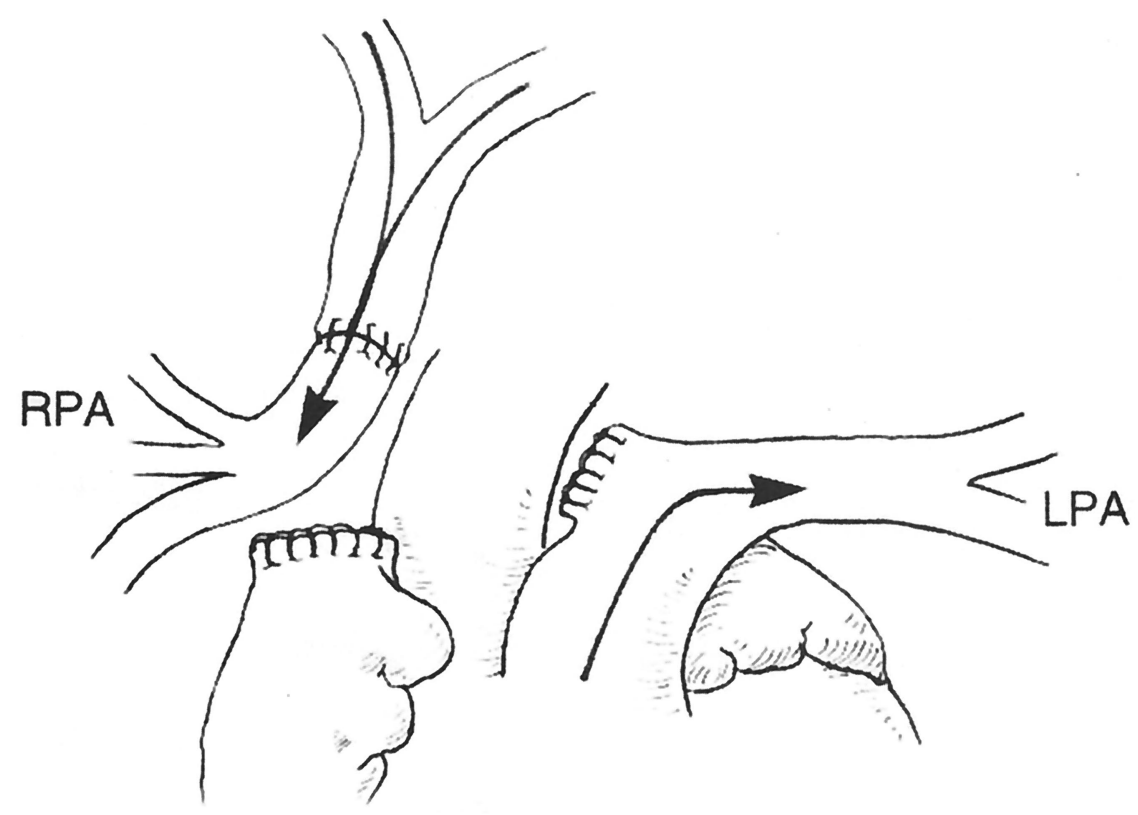

ventilated. After median sternotomy, the lambs were anticoagulated with sodium heparin $(300 \mathrm{U} / \mathrm{kg})$. The lambs were then put to death with an infusion of sodium pentobarbital, the lungs were removed, and samples were snap-frozen in liquid nitrogen.

\section{Western Blot Analysis}

Specimens of right lung tissue from CPA and control groups and left lung tissue from the PAB cohort were obtained as described above. Specimens were homogenized, and proteins were extracted as previously described. ${ }^{10}$ Proteins were then transferred to a polyvinylidene difluoride membrane (Amersham Pharmacia Biotech Inc, Piscataway, NJ). The membrane was soaked in Trisbuffered saline solution $(100 \mathrm{mM}$ Tris- $\mathrm{HCl} \mathrm{pH} 7.5$ and $150 \mathrm{mM}$ $\mathrm{NaCl}$ ) containing 5\% nonfat powdered milk and $0.1 \%$ Tween-20 to block nonspecific binding. The membrane was then incubated overnight at $4{ }^{\circ} \mathrm{C}$ with the following primary antisera at the specified dilutions: HIF- $1 \alpha, 1: 1000$ (BD Transduction Laboratories, Lexington, Ky); VEGF, 1:200 (Santa Cruz Biotechnology, Santa Cruz, Calif); GLUT-1, 1:200 (Flt, Santa Cruz Biotechnology); HO-1, 1:200 (Santa Cruz Biotechnology); and CD62, 1:200 (Research Diagnostics, Inc, Flanders, NJ). Blots were washed and incubated for 30 minutes at $4^{\circ} \mathrm{C}$ with a peroxidase-conjugated secondary antibody (1:20,000 dilution, Vector Laboratories, Inc, Burlingame, Calif). Immunoreactivity was detected with the Supersignal chemiluminescent substrate kit (Pierce Chemical Company, Rockford, Ill). Quantitative assessment of band densities was performed by using scanning densitometry (1D Image Analysis Software; Eastman Kodak Company, Rochester, NY).

\section{Immunohistochemistry}

Immunolocalization was performed as previously described. ${ }^{9}$ In brief, lung tissue from experimental and control animals at 5 weeks after CPA, PAB, or sham surgery was fixed in Histochoice (Amresco, Inc, Solon, Ohio). Tissue was then paraffin embedded, sectioned at $7 \mu \mathrm{m}$, and mounted onto glass slides. Slides were deparaffinized by means of immersion for 6 minutes each in xylene and $100 \%, 95 \%, 70 \%$, and 50\% ethanol. Slides were washed with Tris-buffered saline solution $(50 \mathrm{mmol} / \mathrm{L}, \mathrm{pH} 7.6)$ and then incubated with $0.5 \%$ hydrogen peroxidase with $1 \mathrm{mg} / \mathrm{mL}$ of saponin for 30 minutes to quench endogenous peroxidases. Sections were incubated with $4 \%$ serum from the host species for the secondary antisera to reduce nonspecific antibody binding. Sections were incubated for 18 hours with a 1:200 dilution of either the CD62 or VEGF primary antisera described above. Visualization of staining was accomplished with the Vectastain elite kit (Vector Laboratories), with diaminobenzidine as the chromogen (DAB kit, Zymed Laboratories, San Francisco, Calif).

\section{Statistical Analysis}

Data were analyzed with Statview, a commercially available software package (SAS Institute, Inc, Cary, NC). Protein analysis was evaluated with 3 animals at both 2 and 5 weeks in the CPA, PAB, and sham groups for a total of 6 animals in each cohort. Figures include representative Western blot results for each experimental group. Analysis of statistical differences between groups at each time point was performed by means of paired, 2-tailed Student $t$ tests. Significant differences between the experimental and control groups were confirmed by means of analysis of variance. All values are expressed as means $\pm \mathrm{SD}$.

\section{Results}

Detection of Pulmonary Arteriovenous Shunting Contrast echocardiography detected arteriovenous shunting in all animals evaluated 8 weeks or later $(n=23)$ after CPA. Arteriovenous shunting was absent in the PAB lambs 12 weeks after surgical intervention $(n=3)$. No shunting was detected in any animals studied after sham surgery. Atrial or 

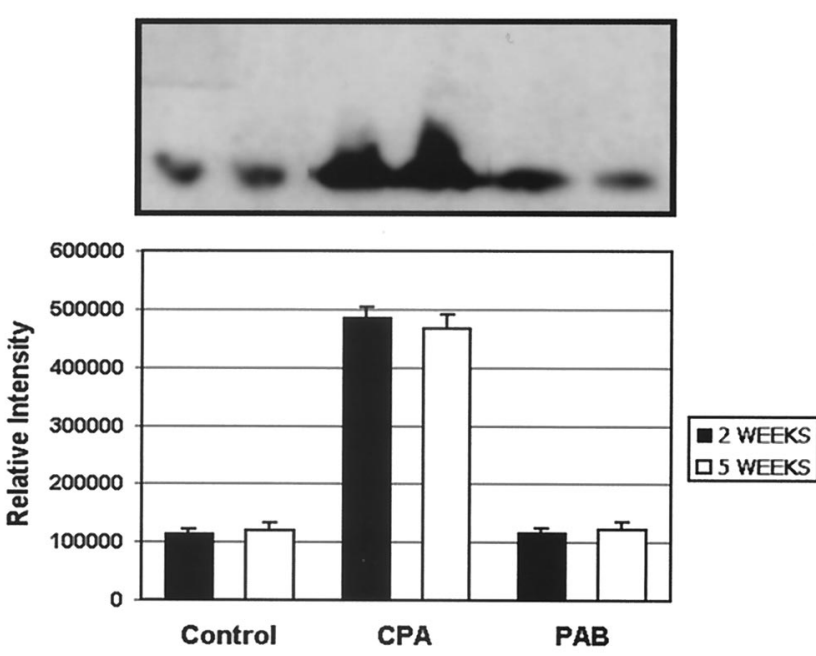

Figure 2. Pulmonary HIF-1 $\alpha$ protein expression after CPA and PAB. Representative results from Western blot analysis are displayed with corresponding densitometry results. Expression increased 4-fold after CPA and was unchanged after PAB. Data are expressed as means $\pm S D$ ( $n=3$ at each time point).
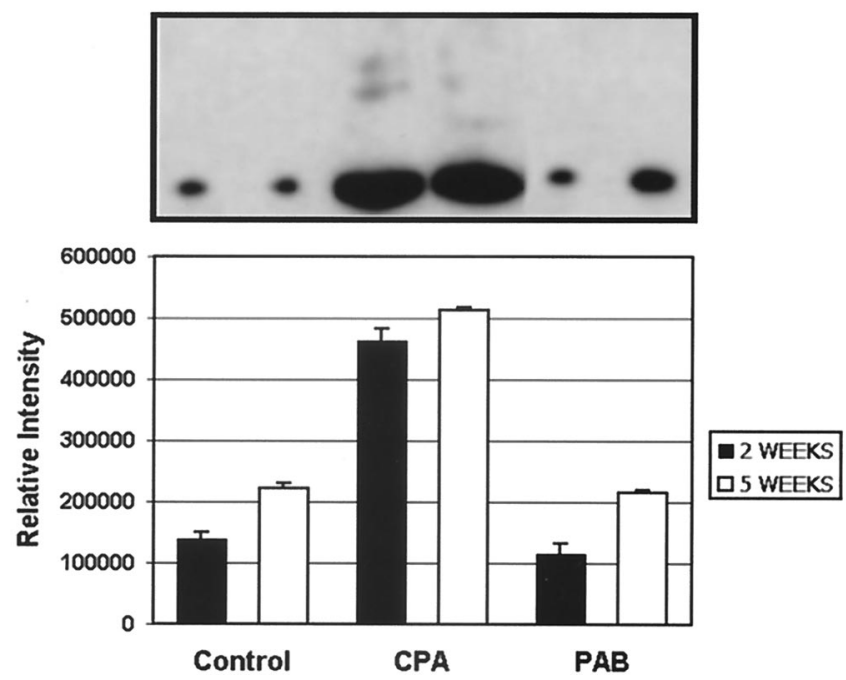

Figure 3. H0-1 protein expression was upregulated 2.5-fold after CPA $(P=.002)$ and unchanged after PAB. Representative results from Western blot analysis are displayed with corresponding densitometry results. Data are expressed as means \pm SD $(n=3$ at each time point).

ventricular septal defects were not present in any of the animals.

\section{HIF-1 $\alpha$ Protein Expression After CPA and PAB}

HIF- $1 \alpha$ was upregulated in lung tissue $4.3 \pm 0.10$-fold $(P<$ $.001)$ at 2 weeks and increased $3.9 \pm 0.16$-fold at 5 weeks $(P=.003)$ after CPA. PAB failed to induce increased

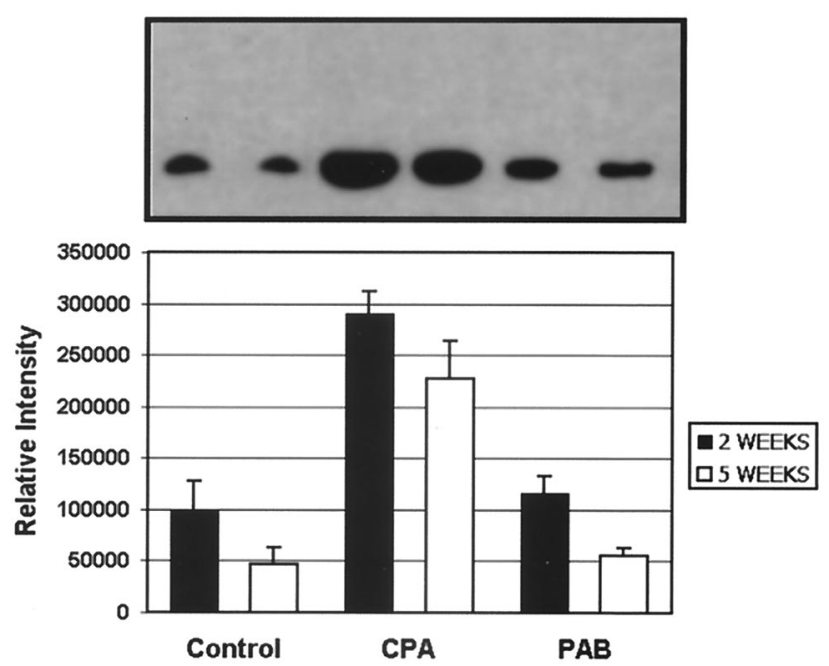

Figure 4. Pulmonary GLUT-1 expression was upregulated 2.5-fold after CPA $(P=.02)$ but was unchanged after PAB. Representative results from Western blot analysis are displayed with corresponding densitometry results. Data are expressed as means \pm SD ( $\mathbf{n}=\mathbf{3}$ at each time point).

HIF- $1 \alpha$ protein expression at either 2 or 5 weeks after surgical intervention $(P>.2$, Figure 2$)$.

Stress-related Gene Expression Was Induced by CPA but not by PAB

Expression of HO-1 and GLUT-1 protein was increased after CPA but not after PAB. HO-1 protein expression was increased $3.3 \pm 0.10$-fold at 2 weeks $(P=.002)$ and $2.3 \pm$ 0.03 -fold at 5 weeks $(P<.001)$ after CPA (Figure 3). CPA caused a $2.5 \pm 0.25$-fold increase in GLUT-1 expression at 2 weeks $(P=.02)$ and a $4.9 \pm 0.58$-fold increase at 5 weeks $(P=.03)$ after the operation (Figure 4$)$. PAB did not result in a statistically significant increase in the expression of HO-1 or GLUT-1 at either time point $(P>.2)$.

\section{CD62 Expression Was Induced Only After CPA}

CPA caused a marked increase in the expression of CD62, a marker of endothelial activation. There was a $4.6 \pm$ 0.1 -fold increase at 2 weeks $(P=.002)$ and a $3.6 \pm$ 0.02 -fold increase at 5 weeks in CD62 expression $(P<$ $.001)$. CD62 expression was unchanged at both 2 and 5 weeks after PAB $(P>.2)$ (Figure 5).

CPA and PAB Both Cause Enhanced Pulmonary Angiogenic Signaling

Pulmonary expression of VEGF and Flt-1, a VEGF receptor, were enhanced after both procedures. VEGF expression was increased $4.9 \pm 0.36$-fold in CPA specimens $(P=$ $.002)$ and $3.7 \pm 0.52$-fold in PAB specimens $(P=.004)$ at 2 weeks. At 5 weeks, expression of VEGF protein was upregulated $3.5 \pm 0.16$-fold after CPA $(P=.003)$ and 


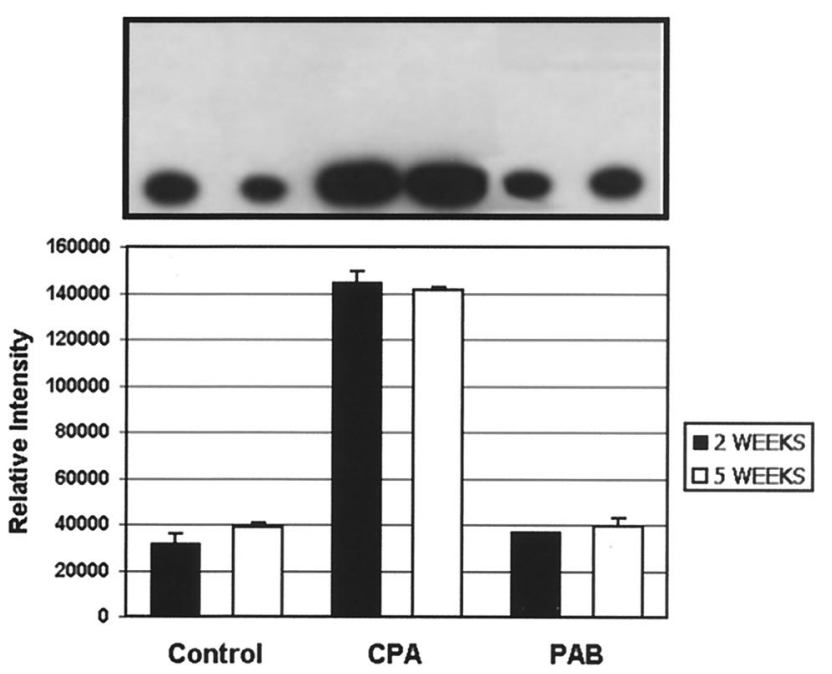

Figure 5. Pulmonary CD62 protein expression was upregulated 3to 4-fold after CPA ( $P=.002)$ and unchanged after PAB. Representative results from Western blot analysis are displayed with corresponding densitometry results. Data are expressed as means \pm SD ( $n=3$ at each time point).

$3.2 \pm 0.26$-fold after PAB $(P=.01$, Figure 6$)$. Pulmonary flt-1 protein expression was upregulated $2.5 \pm 0.28$-fold in CPA specimens $(P=.02)$ and $2.1 \pm 0.26$-fold in PAB specimens $(P=.03) 2$ weeks after the operation. At 5 weeks, flt-1 expression was increased $3.4 \pm 0.19$-fold after CPA $(P=.006)$ and $3.5 \pm 0.21$-fold after PAB $(P=.006$, Figure 7).

\section{Immunolocalization of VEGF to Pulmonary \\ Vasculature}

At 5 weeks, immunohistochemistry of lung sections revealed that CPA and PAB both enhanced endothelial staining of VEGF when compared with the sham control specimens (Figure 8). In the control specimens pulmonary VEGF staining was not remarkable in comparison with background levels.

\section{Immunolocalization of P-Selectin/CD62 to Pulmonary Vasculature \\ Marked CD62 staining of pulmonary arterial endothelium was present at 5 weeks after CPA but was absent in both the $\mathrm{PAB}$ and control sections (Figure 9). Time-matched control specimens demonstrated only minimal staining of the pul- monary microvascular endothelium.}

\section{Discussion}

The development of pulmonary AVMs after CPA can have disastrous consequences for the child with cyanotic heart disease, leading to further hemodynamic decompensation and, ultimately, death. The cause of the pulmonary vascular remodeling in this setting is still unknown.

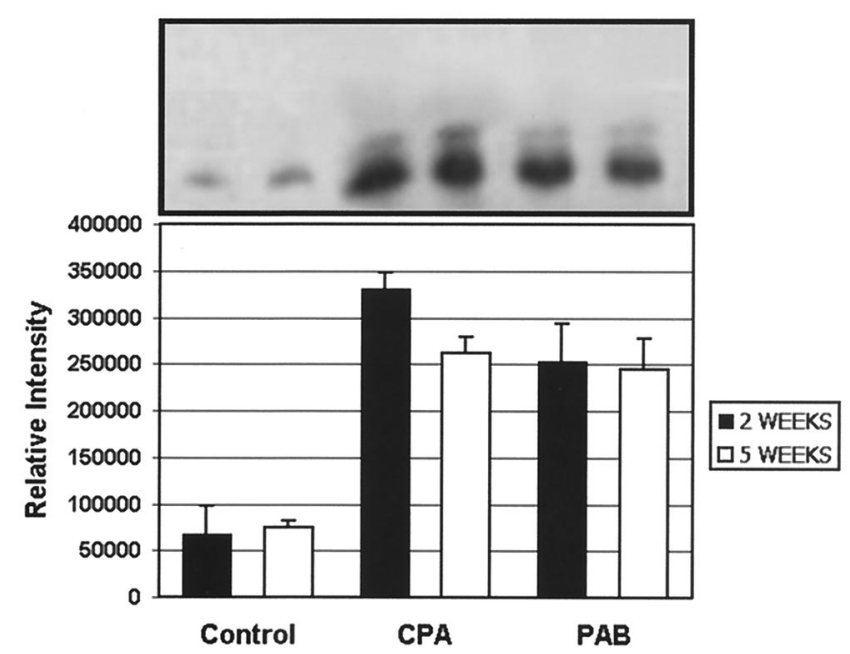

Figure 6. Pulmonary VEGF protein expression was upregulated 3to 4-fold after both CPA $(P=.003)$ and PAB $(P=.008)$. Representative results from Western blot analysis are displayed with corresponding densitometry results. Data are expressed as means $\pm S D$ ( $n=3$ at each time point).

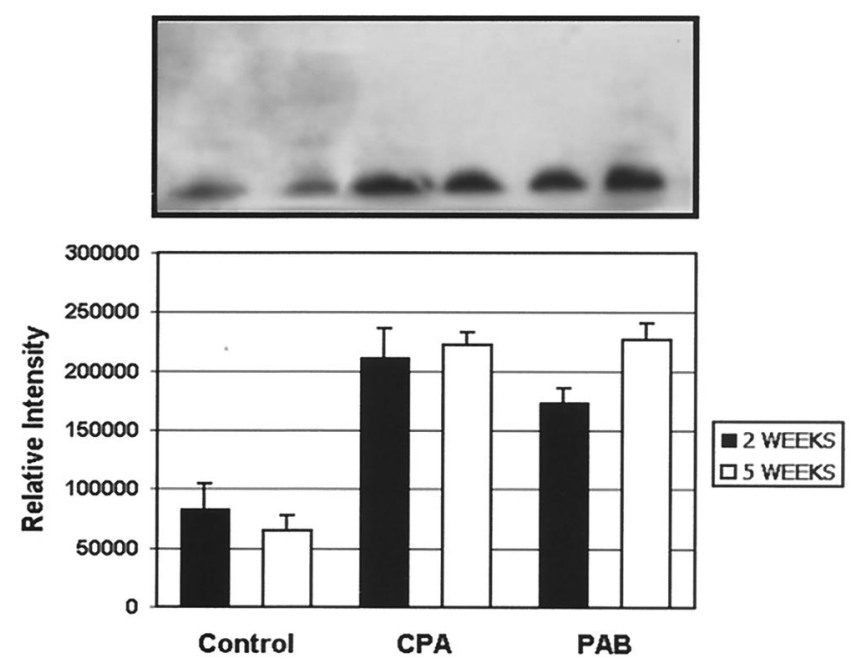

Figure 7. Pulmonary Flt-1 protein expression was increased 2- to 3-fold after CPA $(P=.006)$ and PAB $(P=.01)$. Representative results from Western blot analysis are displayed with corresponding densitometry results. Data are expressed as means \pm SD ( $n=3$ at each time point).

Our results suggest that CPA causes oxidant stress within the pulmonary vasculature. CPA markedly increases the expression of the stress-associated factors, including HIF$1 \alpha$. Moreover, this phenomenon is not limited to a HIF- $1 \alpha-$ specific pathway because CD62 is also upregulated. Enhanced oxidative stress-related signaling was independent of reduced pulmonary blood flow because PAB failed to cause increased expression of any of these genes.

Interestingly, enhanced angiogenic signaling may not 


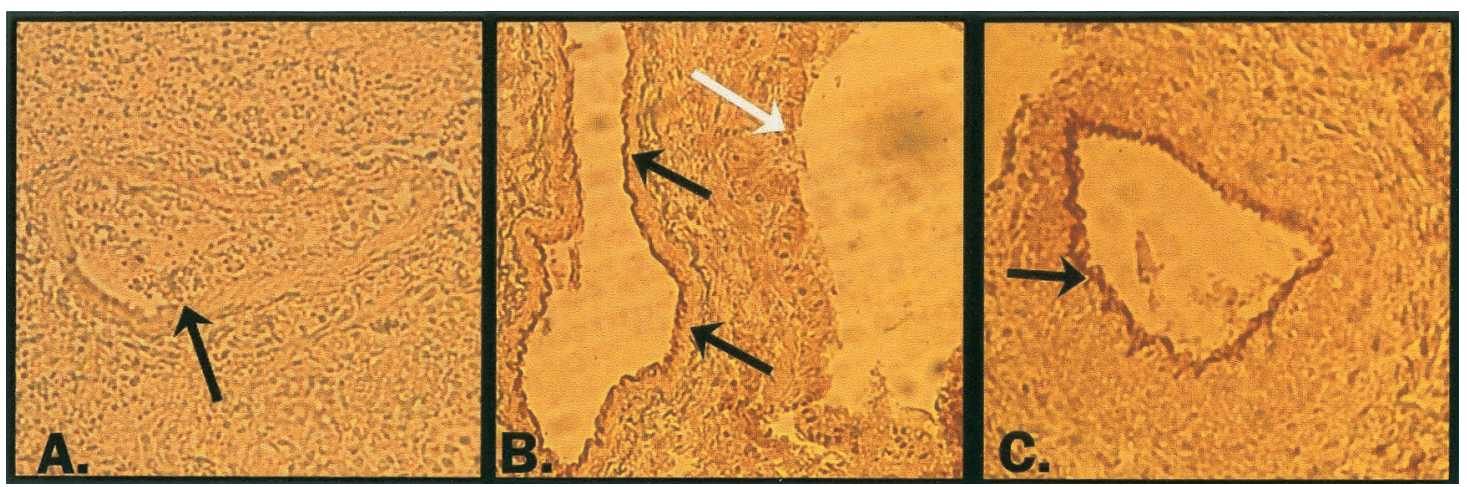

Figure 8. Photomicrographs of VEGF immunolocalization in lung tissue 5 weeks after the operation. (Original magnification $200 \times$.) In A there is a lack of staining in the pulmonary arteriolar wall of the sham-operated control specimens. Enhanced endothelial staining 2 weeks after CPA is demonstrated in B. Black arrows indicate stained endothelium. The white arrow identifies the airway wall for comparison. In C increased staining is demonstrated in PAB specimens as well.

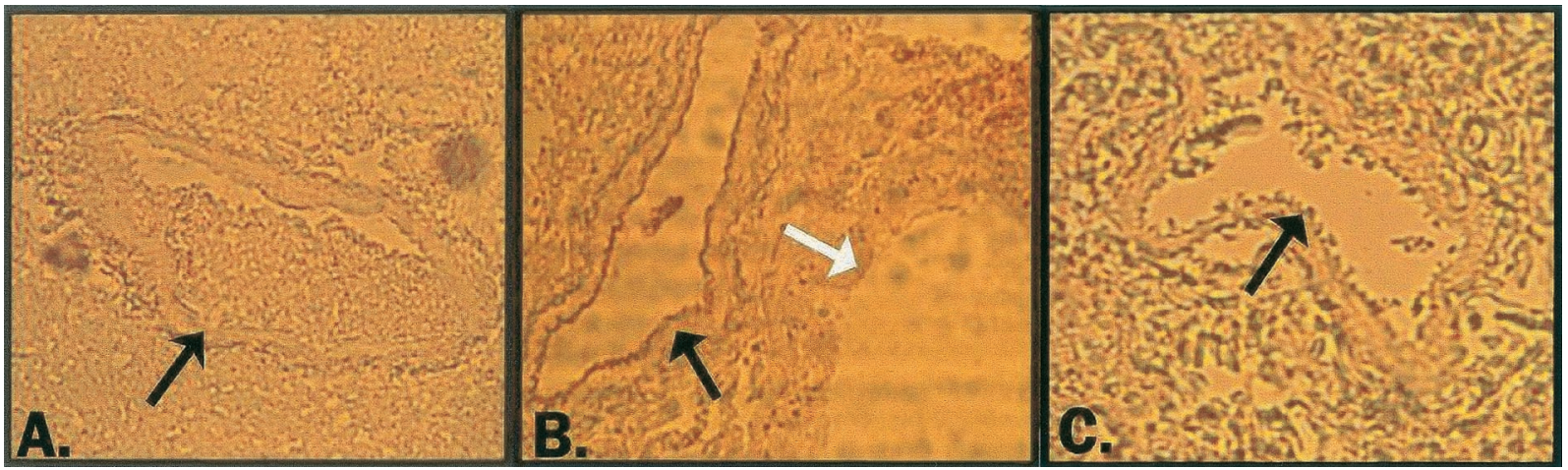

Figure 9. Immunolocalization of CD62. (Original magnification $200 \times$.) Shown are photomicrographs of control $(A)$, CPA (B), and PAB (C) specimens 5 weeks after the operation. In A arrows indicate the unstained vessel wall. In B upregulation of angiotensin II after CPA is localized to pulmonary arterial endothelium. The white arrow indicates unstained bronchiolar wall. The PAB specimen in $\mathrm{C}$ reveals a lack of increased pulmonary endothelial CD62 expression.

play a central role in the formation of pulmonary AVMs because $\mathrm{CPA}$ and $\mathrm{PAB}$ both increase the expression of VEGF and Flt-1. The early appearance of increased subpleural vascular markings after CPA led us to initially suspect the involvement of an angiogenic stimulus in the development of these AVMs. However, we found the same degree of enhanced subpleural vascularity in the lungs of the sheep that underwent PAB. Yet we found no evidence of formation pulmonary AVMs after PAB in this animal model. Presumably, reduced pulmonary blood flow is the stimulus for the increased expression of these angiogenic factors.

The altered physiology resulting from CPA may cause a change in the redox state of the pulmonary vascular bed. Exclusion of inferior venous drainage from the pulmonary circulation could potentially interrupt the delivery of a mediator of normal vascular development derived from the liver or some other lower body source. This theory is supported by reports of resolution of pulmonary AVMs after the inclusion of inferior venous blood. ${ }^{11,12}$ Pulmonary endothelial oxidative stress may result from the lack of this putative protective factor and serve as a stimulus for the pathologic vascular remodeling responsible for arteriovenous shunting. Further work is warranted to fully understand the mechanisms involved in formation of pulmonary AVMs after CPA.

We thank Mario Trujillo and Sam Suleman for their technical assistance.

\section{References}

1. Glenn WWL, Patino JF. Circulatory bypass of the right heart. I. Preliminary observations on the direct delivery of the vena caval blood into the pulmonary arterial circulation: azygos vein-pulmonary artery shunt. Yale J Biol Med. 1954;27:147-51. 
2. Cloutier A, Ash JM, Smallhorn JF, Williams WG, Trusler GA, Rowe $\mathrm{RD}$, et al. Abnormal distribution of pulmonary blood flow after the Glenn shunt or Fontan procedure: risk of development of arteriovenous fistulae. Circulation. 1985;72:471-9.

3. Chang RR, Alejos JC, Atkinson D, Jensen R, Drant S, Galindo A, et al. Bubble contrast echocardiography in detecting pulmonary arteriovenous shunting in children with univentricular heart after cavopulmonary anastomosis. J Am Coll Cardiol. 1999;33:2052-8.

4. Duncan BW, Kneebone JM, Chi EY, Hraksa V, Isik FF, Rosenthal GL, et al. A detailed histological analysis of pulmonary arteriovenous malformations in children with cyanotic congenital heart disease. J Thorac Cardiovasc Surg. 1999;117:931-8.

5. Abrams LD. Side-to-side cavopulmonary anastomosis for the palliation of the "primitive ventricle" [abstract]. Br Heart J. 1077;39:926.

6. Malhotra SP, Riemer RK, Thelitz S, He Y, Hanley FL, Reddy VM. Superior cavopulmonary anastomosis suppresses pulmonary angiotensin-converting enzyme activity and expression. J Thorac Cardiovasc Surg. 2001;122:464-9.

7. Semenza GL. HIF-1 and human disease: one highly involved factor. Genes Dev. 2000;14:1983-91.

8. Semenza GL. Expression of hypoxia-inducible factor 1: mechanisms and consequences. Biochem Pharmacol. 2000;59:47-53.

9. Bernstein HS, Brook MM, Silverman NH, Bristow J. Development of pulmonary arteriovenous fistulae in children after cavopulmonary shunt. Circulation. 1995;92:309-14.

10. Riemer RK, Buscher C, Bansal RK, Black SM, He Y, Natuzzi E. Increased expression of nitric oxide synthase in the myometrium of the pregnant rat uterus. Am J Physiol. 1997;272:E1008-15.

11. Srivastava D, Preminger T, Lock JE, Mandell V, Keane JF, Mayer JE, et al. Hepatic venous blood and the development of pulmonary arteriovenous malformations in congenital heart disease. Circulation. 1995;92:1217-22.

12. Shah MJ, Rychik J, Fogel MA, Murphy JD, Jacobs ML. Pulmonary AV malformations after cavopulmonary connection: resolution after inclusion of hepatic veins in the pulmonary circulation. Ann Thorac Surg. 1997;63:960-3.

\section{Discussion}

Dr Yasunaru Kawashima (Osaka, Japan). As one of the criminals of producing pulmonary AVMs by advocating the total cavopulmonary shunt operation, I am very much grateful to the authors for investigating the reasons for pulmonary AVMs. I understand, from your presentation, that you have shown that CPA, rather than PAB, upregulated the oxidative stress. I wonder how you can imagine that the oxidative stress may predispose the affected vasculature to arteriovenous shunting.

It is my question whether the oxidative stress in patients with well-developed AVM will upregulate the oxidative stress. Another question is whether you think that the upregulated oxidative stress is a fundamental factor in addition to the lack of some substances delivered from the liver and perfused into the lung in development of pulmonary AVMs.
Dr Malhotra. To the first question, oxidative stress probably affects various endothelium-dependent vasodilation mechanisms and probably inhibits regulation of normal vascular tone. I believe it does create a substrate, perhaps for the development of pulmonary AVMs. Unfortunately, I think we still have much work to do on this topic.

Dr Frank Sellke (Boston, Mass). Your hypothesis is that there is a differential expression of these growth factors, such as HIF$1 \alpha$, and that this is based on oxidative stress. You can measure oxidative stress in the different lung segments. That would really pin down your hypothesis. Have you done that or are you considering doing that?

Dr Malhotra. We have not done that at this time, but that would be an interesting investigation to pursue.

Dr Sellke. Also, I believe you showed that expression of VEGF was increased in the vessel, as opposed to the surrounding tissue.

Dr Malhotra. We have had localization to the endothelium, yes.

Dr Sellke. The VEGF receptors are localized to the endothelium, but VEGF expression is usually increased everywhere. I think that is true in the lung, in the heart, and in skeletal muscle. I am surprised that the expression in your study was localized to the vessel wall. Is that what you found?

Dr Malhotra. Right, that the protein is increased in the vessel wall.

Dr Christian Pizarro (Orlando, Fla). If these animals, after you created the model, were noncyanotic, how do you put together the fact that HIF was induced when I would take it that the saturations were over $90 \%$ ?

Second, did you look at EGR-1 and the theory that early growth factor is supposed to be induced as rapidly as HIF-1 in patients who have this type of model created?

Third, did you look at different isoforms of VEGF, meaning the short versus the long forms? We know that they are located and express differently, and perhaps you could have looked at some of that.

Dr Malhotra. To explain upregulation of HIF, we do not postulate that it is a hypoxic environment. These lambs were normal healthy lambs that underwent CPA. We do, however, believe that HIF is a detector of redox status in the parenchyma, and the redox status may be altered by the lack of this hepatic factor.

Second, in response to early growth factor, we have just begun to look at that, and therefore that is a very timely point.

In terms of the isoforms, we have only looked at VEGF-165. 